Rev. Elev, Méd. véf. Poys trop., 1968, 21, 1 (103-112).

\title{
Composition en acides-aminés des principales légumineuses fourragères de Madagascar
}

\author{
par R. GAULIER (*) \\ ovec la collaboration technique de $M^{\text {me }}$ LAURON
}

\begin{abstract}
RÉSUMÉ
La composition en acides-aminés des princıpales légumineuses fourragères de Madagascar a été déterminée après hydrolyse, séparation par chromatographie sur colonne de résine, et réaction colorée à la ninhydrine.

Le tryptophane a été dosé selon la méthode colorimétrique de Fischl.

Les légumineuses fourragères suivantes ont été analysées :

- Vigna sinensis ;

- Soja hispida ;

- Stylosanthes gracilis;

- Pueraria phoseolides:

- Pueraria Thunbergiana;

- Desmodium intortum :

- Phoseolus atropurpureus:

- Centrosema pubescens ;

- Giycine javanica:

- Albizzio lebbek.
\end{abstract}

Les résultats sont présentés sous forme de tableaux.

\section{INTRODUCTION}

L'apport d'aliments azotés pose un grave problème pour l'Elevage d̀ Madagascar. Les pâturages naturels y sont de types tropicaux, constifués surtout par des gramınées, et sont généralement pauvres en légumineuses.

Leurs teneurs en protéines sont par suite relativement faibles et cet inconvénient est aggravé par le fait qu'après une flambée de végétation correspondant à la première moltıé de la saison des pluies, ces plantes sèchent rapldement, et leur taux de protides diminue encore (8).

Pour ces raisons l'apport d'un complément azoté dans l'alımentatıon des animaux est ındis-

(*) Pharmacien-Chımiste Lieutenant-Coionel. pensable. Il s'effectue essentiellement à Madagascar sous forme de tourteaux d'arachide dont la teneur en protides esł de l'ordre de 40 à 45 p. 100.

Malheureusement, les graines d'arachides traitées sont souvent contaminées par l'Aspergillus flovus et les tourteaux obtenus contiennent de l'Aflatoxine qui leur confère une toxicité généralement faible mais qui, dans quelques cas, peut être importante.

Comme autres compléments protidiques, l'éleveur dispose également, mais de façon irrégulière, de quelques produits locaux d'origine animale, dont la farine de sang et la farine de viande.

Citons également les issues de riz et le maìs, qui procurent un apport moyen de protides dans l'alimentation. 
Nous voyons done que, pour remédier à lo pauvreté protidique des pâturages naturels, il n'existe à Madagascar qu'un nombre très restreint d'aliments riches en azote. L'utilisation ef l'extension des cultures de légumineuses fourragères sont susceptibles d'apporter une notable amélioration à ce problème.

Ces légumineuses ont, en effet, une teneur en protides généralement très supérieure à celle des graminées, et elles présentent en outre l'avantage de pouvoir être consommées à l'état de fourrage vert pendant une plus grande période de l'année. Enfin, par leur richesse en sels minéraux, les légumineuses contribuent également à suppléer à la carence des graminées en calcium et en phosphore.

L'intérêt des légumineuses fourragères ne se limite pas à leur utilisation sous forme de fourrage vert ou de foin. Séchées ef broyées, la plupart d'entre elles fournissent en effet des forines riches en protides, pouvant être substituées, au moins en partie, aux quelques produits azotés de base cités ci-dessus, pour la préparation de provendes, apportant ainsi une plus grande variété dans l'alimentatıon des divers anımaux d'élevage.

C'est ainsi que la multiplication des cultures de légumineuses fourragères est susceptıble de fournir à l'important élevage bovın de Madagascar (quelque 9 millions de têtes) un apport quantitatif très appréciable de matières azotées.

Les avantages des légumineuses fourragères ne sont pas moins grands dans la nutrition des porcs dont l'élevage se pratique surtout sur les Hauts-Plateaux. Leurs besoins en protides sont importants et l'utılisation des légumineuses est d'autant plus intéressante que le pâturage direct constitue un mode économique d'alimentation pour les porcs.

Signalons enfin l'utilisation des légumineuses fourragères dans l'alımentation des volailles, et en particulier des oies et des canards qui sont de gros consommateurs de verdure, et dont l'élevage à Madagascar est en voie d'expansion.

Si pour les bovins - capables du fait de la conformation de leur appareil digestif et de leur flore intestinale, de faire la synthèse de leurs acides-aminés - l'apport d'aliments azotés ne pose qu'un problème quantitatif, il n'en est plus de même pour les porcs et les volailies qui doivent trouver dans leur alimentation qualitative- ment et quantitativement les acides-cmınés dont leur organisme a besoin.

Il est donc important de connaître la teneur en amino-acides des protides entrant dans leur alimentation.

C'est dans ce but que nous avons déterminé la composition en acides-aminés des principales légumıneuses fourragères qui sont actuellement à la disposition de l'élevage de Madagascar et qui sont, en particulier, susceptibles d'entrer dans la préparation des provendes destinées à l'alimentation des porcs et des volailles.

\section{PRINCIPALES LÉGUMINEUSES FOURRAGÈRES DE MADAGASCAR}

La légumineuse fourragère-type des climats tempérés est la luzerne. Mais celle-ci s'adapte mal aux terrains acides qui constituent la majorité des sols de Madagascar (8). Aussi a-t-on été conduit à utilıser d'autres espèces mieux adaptées aux sols ef aux climats tropicaux. De nombreux essais ont aınsi été effectués, souvent avec succès, à Madagascar, dans le but d'améliorer les pâturages naturels, ou de créer des pâturages artificiels, solt avec des légumineuses pures, soit avec des associations de légumineuses et de graminées.

Parmi ces légumıneuses fourragères, deux sont des plantes annuelles : le Vigna sinensis et le Soja.

Les autres sont des légumineuses pérennes.

\section{Vigna sinensis (Voanemba-Vohem).}

Le Vigna sinensis est cultivé de longue date d̀ Madagascar, où sa graine est utilisée dans l'alimentation humaine. Il est également très employé comme engrais vert. Enfin, il fournit un fourrage excellent en vert comme en forn, et la farine de Vigna simensis donne de bons résultats dans les provendes destinées aux volailles (10).

\section{Soja hispida ou glycine soja (Soja).}

Déjà important par son rôle dans l'alimentation humaine où ses graines son très utilisées, le Soja constitue également un fourrage très riche. Fané et broyé, le Soja fournit une poudre qui entre dans la composition de provendes destinées aux volailles et aux porcs (8). 


\section{Stylosonthes gracilis.}

Introduit à Madagascar en 1953 comme plante de couverture, engrais vert et plante fourragère, le Stylosanthes gracilis donne de très bons résuliats. Il est cultivé soit en culture pure, soit en association avec des graminées, pour constituer des prairies de pâture ou de fauche. II donne également de bons résultats dans l'amélıoration des pâturages naturels (3). II fournit en outre une farine de bonne qualité, entrant dans la composition des provendes.

\section{Pueraria phaseolides (Kudzu).}

L'appellation «KUDZU » intéresse différentes espèces de Pueraria. A Madagascar, ce terme s'applique plus spécialement au Pueraria phaseolides. La culture de cette légumineuse donne de très bons résultats dans les régions où l'altitude ne dépasse pas $1000 \mathrm{~m}$, et en partıculier dans les régions côtières (température élevée). Utılisé d'abord comme plante de couverture et engrais vert, le Kudzu s'est révélé comme étant l'un des meilleurs fourrages verts utilisables en fin de saison sèche, soit en pâturage par les bovins et les porcs, soit pour affouragement (2).

Cette légumineuse sert également à préparer une farine destinée d̀ être incorporée aux provendes des bovins, des porcs et des volailles.

\section{Pueraria thunbergiana.}

Le Puerario thumbergiona donne en particulier de bons résultats dans les régions où l'altıtude dépasse $1.000 \mathrm{~m}$ (Hauts-Plateaux), où II remplace le Puerario phaseolides.

II se présente surtout à l'état de culture pure, et il est bien appété par les animaux.

\section{Desmodium infortum.}

La culture de cette légumineuse fourragère, qui en est encore au stade expérımental, donne des résultats satisfaisants dans les régions de moyenne altitude et sur les Plajeaux. Le Desmodium intortum est surtout utilisé en vert ou en foin pour affouragement à l'étable (8).

\section{Phaseolus afropurpureus.}

Récemment introduite à Madagascar (1963), cette légumineuse donne des résultats très encou- rageants par son adapiation à la sécheresse, sa résıstance au pâturage ef au piétinement, ainsi que par sa productivité élevée. Elle est susceptible de donner d'excellents résultats en association avec les graminées.

\section{Centrosemo pubescens.}

Il est cultivé soit pour améliorer le pâturage naturel, soit pour implantation d'un pâturage artificiel en association avec des graminées.

\section{Glycine jovonica.}

Cette légumıneuse est avant tout une plante de couverture et donne de bons résultats dans la conservation des sols. Ce n'est qu'accessoirement que le Glycine jovanica est employé comme plante fourrogère.

Il faut enfin citer à part :

\section{L'albizzia lebbek ou Bonara.}

C'est un arbre de la famille des légumineuses, communément répandu dans les régions côtières de Madagascar, ou son fevillage vert, lorsqu'il est exploité par l'homme, fournit un appoint très apprécıé du bétail ( 8 ).

\section{ÉTUDE BIOCHIMIQUE}

Cette étude a porté :

10 Sur la composition brochimique globale.

Les techniques utilisées sont celles décrites dans la «Mise à jour au 1er juin 1961 des méthodes officielles employées par les Laboratoires du Service de la Répression des Fraudes pour l'analyse des échantıllons de produits de l'alimentation anımale ».

Laboratoire d'Alimentation de l'Ecole Nationale Vétérinaire d'Alfort, Fascicule XXVI, BOSC Frères, Editeurs, Lyon, 1961.

Les résultats figurent au tableau No 1.

\section{Sur la composition en ocides-ominés.}

Les acıdes-aminés, libérés par une hydrolyse acide de 24 heures dans un premier essal ef de 30 heures dans un $2^{2}$ essai, ont été séparés par chromatographie sur colonne de résine « Chromo-Beads, Type A» de TECHNICON, selon la 


\begin{tabular}{|c|c|c|c|c|c|c|c|c|c|c|}
\hline $976^{\circ} 0$ & $\angle 68^{\circ} 0$ & $76 L^{\circ} 0$ & $786^{\circ} 0$ & $0<9^{\circ} \circ$ & $S \in Z “ I$ & $\angle 89^{\circ} \mathrm{O}$ & カカグ $\mathrm{L}$ & $020^{\circ} \mathrm{T}$ & $86 Z^{*} \mathrm{I}$ & 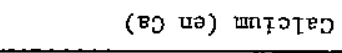 \\
\hline$I \mapsto Z^{\prime} 0$ & $98 \tau^{c} 0$ & $70 z^{c} 0$ & $70 \varepsilon^{c} 0$ & $8 \mathrm{t} \tau^{6} 0$ & $9+\tau^{\circ} 0$ & $78 \mathrm{~T}^{\circ} \mathrm{O}$ & $\angle 9 I^{\circ} O$ & $68 I^{\prime} 0$ & $525^{\circ} 0$ & (d uə) әxочฺdsoud \\
\hline II'O & $88^{\circ} 0$ & $8 L^{\circ} \mathrm{I}$ & $75^{\circ} 0$ & 加“ I & $\mathrm{L} Z^{\star} \mathrm{\tau}$ & $\dagger z^{\mathbf{6}} \dot{\varepsilon}$ & $8 \varepsilon^{\circ} 0$ & $95^{6} \mathrm{~T}$ & $\angle 9^{\circ} \mathrm{O}$ & 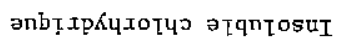 \\
\hline & \multicolumn{10}{|c|}{ 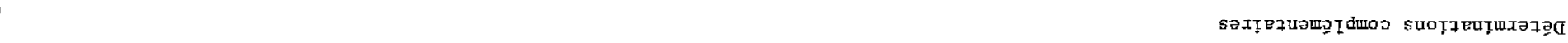 } \\
\hline$I E^{\prime} \angle \varepsilon$ & $6 S^{6} \angle E$ & $6 Z^{\circ} \angle \varepsilon$ & $58^{*} 7 \varepsilon$ & $6 L^{\circ} \mathrm{OH}$ & $98^{\circ} T^{\dagger}$ & $\tau \angle C \angle H$ & $\varepsilon Z^{\prime} \tau$ & $8 z^{\prime}<\varepsilon$ & {$\left[\begin{array}{l}4 \\
4\end{array}\right.$} & 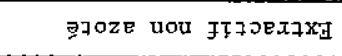 \\
\hline I8 $O E$ & $\angle 0^{\circ} 7 \varepsilon$ & $50^{c} \varepsilon \varepsilon$ & $O \tau^{c} \tau \varepsilon$ & $80^{\circ} \varepsilon E$ & $58^{\circ} \pitchfork \varepsilon$ & $E \rightarrow L Z$ & $\eta L^{\prime} \varepsilon \varepsilon$ & $\varepsilon \eta^{\prime} 9 z$ & $68^{\circ} \mathrm{Tt}$ & ałnבq อsoโnโtวอ \\
\hline $20^{\circ} \mathrm{Lz}$ & $6 I ‘ 8 I$ & $08^{6} 8 \mathrm{~L}$ & $65^{\circ} \mathrm{OZ}$ & $\angle Z \cdot 9 \tau$ & $S I^{\prime} \varepsilon \tau$ & $29^{\circ} Z \mathrm{I}$ & $S S^{\prime} S \tau$ & $0 s^{c} z z$ & $\varepsilon 0^{*} \nabla z$ & 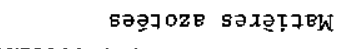 \\
\hline $68^{\circ} 7$ & $\pi z^{\prime} z$ & $\eta I^{\prime} \varepsilon$ & $60^{4}+7$ & $20^{\circ} \mathrm{Z}$ & $8 z^{\circ} \mathrm{Z}$ & $O H^{6} \varepsilon$ & $\varsigma 6^{\circ} \mathrm{L}$ & $\operatorname{LH}^{\circ} \varepsilon$ & $\angle 7^{*} 9$ & 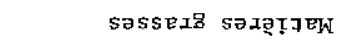 \\
\hline$\angle 6^{\circ} \mathrm{s}$ & $16^{\circ} \mathrm{L}$ & $Z L \angle$ & $L Z \times 8$ & $38^{\circ} L$ & $98^{\circ} \mathrm{L}$ & $78 \div 8$ & $\varepsilon 5^{c} L$ & $Z \varepsilon^{\circ} O T$ & $0 z^{\star} 6$ & 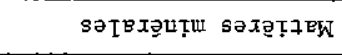 \\
\hline $\begin{array}{c}\text { yaqqa2 } \\
\text { p2zz?q2V }\end{array}$ & $\begin{array}{l}\text { Doubare } \\
\text { as } 20 h 25\end{array}$ & $\begin{array}{l}\text { subosaqnd } \\
\text { butasouquas }\end{array}$ & $\begin{array}{c}\text { snarnderndoxpp } \\
\text { sn Zoasmld }\end{array}$ & $\begin{array}{l}\text { unz.xołu? } \\
\text { unzpousag }\end{array}$ & 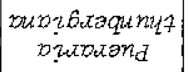 & 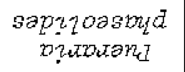 & $\begin{array}{c}\text { s.2.20nth } \\
\text { sauzupsozhas }\end{array}$ & $\begin{array}{c}m ? d s u \\
p^{2} \circ{ }^{2}\end{array}$ & $\begin{array}{l}\text { s.suburs } \\
\text { an } 621\end{array}$ & 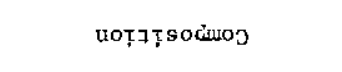 \\
\hline
\end{tabular}

(כอs 7xnpord ap oot Inod)

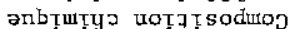

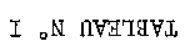




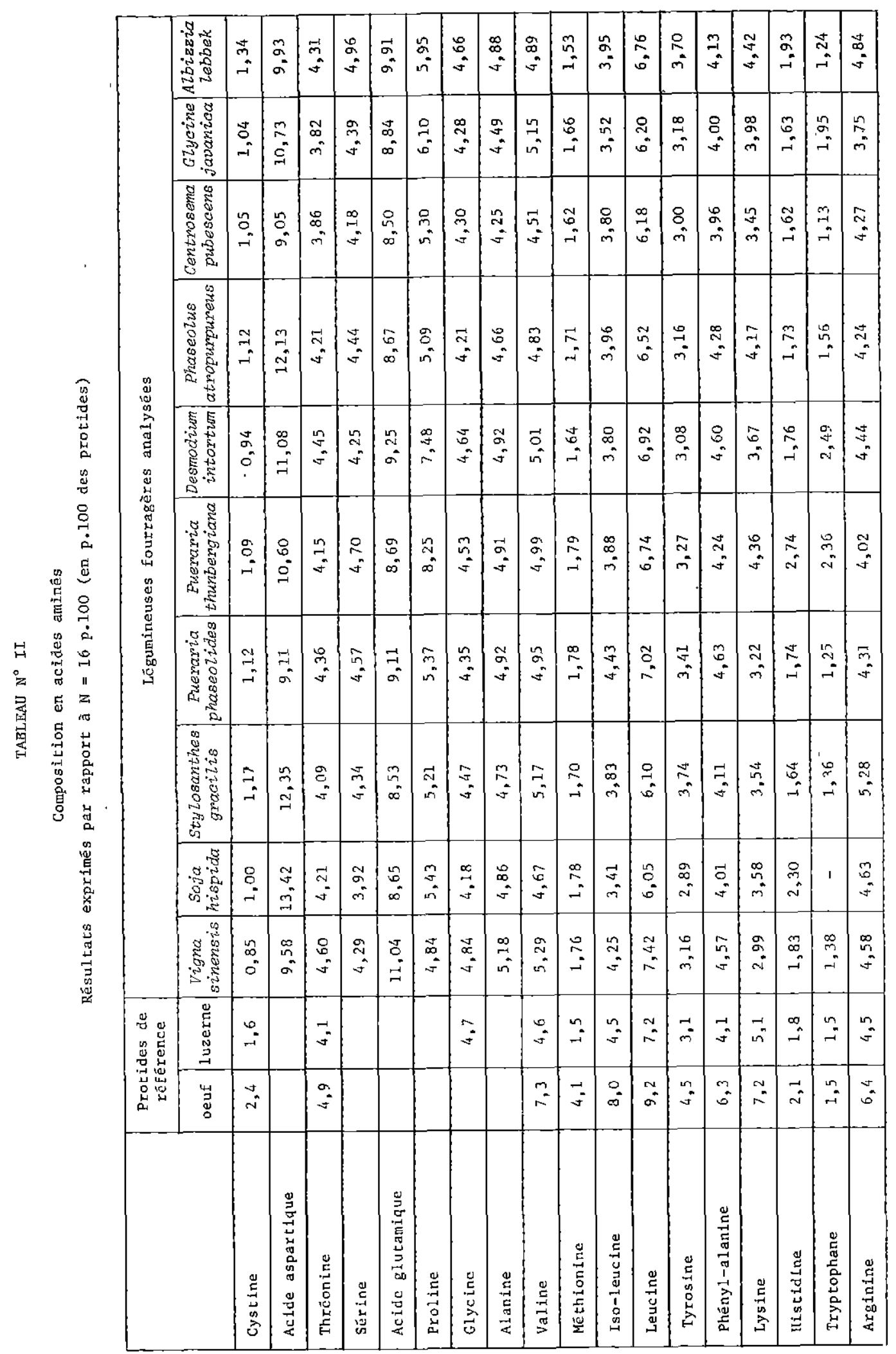


méthode de MOORE, SPACKMAN ef STEIN ( 5 et 6 ) et dosés par le réactif à la ninhydrine de MOORE et STEIN (7).

La cystine est déterminée quantitativement sous forme d'acide cystéique par la méthode de SCHRAM, MOORE et BIGWOOD (9).

Le tryptophane est dosé après hydrolyse alcaline par la réaction de FISCHL, modifiée par INGLIS et LEAVER (4).

Nos résultats figurent dans le Tableau !l.

Pour apprécier la valeur nutritive des protides étudiés, nous avons inscrit dans ces Tableaux la composition en acides-aminés des protides de l'œuf, pris comme étalon de référence, selon la méthode de MITCHELL et BLOCK (1). On sait que, pour chaque acide-aminé, la comparaison par rapport à cet élément de référence se traduit par une différence exprimée en pourcentage.

II nous a paru intéressant également d'indiquer la composition en acides-aminés des protides d'une luzerne des pays tempérés, pour la comparer avec les légumineuses étudiées. Les différences peuvent s'exprimer en pourcentage, de la même façon que ci-dessus : nous en donnons plus loin quelques valeurs.

\section{Matériel végétal utilisé.}

Nos analyses ont porté sur des échantillons (un par espèce végétale) correspondant à des fourrages prêts à être consommés par les animaux (en tenant compte du stade de végétation, des partıes consommables, des conditions de fanage, etc...).

Nous donnons cl-après les renseignements concernant chaque prélèvement. II convient de signaler que certaıns échantillons provenant des Centres de Recherches Zootechniques de Kianjasoa et de Miadana ont été cultivés sur un sol ayant reçu une forte fumure organıque $(30 \mathrm{t}$ de fumier à l'hectare) et que ces apports importants de fumure - habituels dans ces centres expérimentaux - sont exceptionnels dans la pratique de la culture coutumière à Madagascar.

\section{Vigna sinensis.}

Provenance :

Région de Tananarive.
Culture:

Sur colline.

Fumure :

Minérale ef organique.

Prélèvement :

Parties aériennes de la plante (feuilles et tiges), à la préfloraison.

\section{Soja hispida (variété à grains blancs).}

Provenance :

Centre de Recherches Zootechniques de Kianjasoa.

Cultivé :

Sur sol ferrallitique de plateau, de valeur moyenne.

Fumure :

Organique ( 30 t de fumier à l'hectare).

Prélèvement :

Parties aériennes de la plante fevilles ef tiges) à la préfloraison.

\section{Stylosanthes gracilis.}

Provenance :

Centre de Recherches Zootechniques de Kianjasoa.

Nature dusol:

Sol ferrallitique de plateau, "de valeur moyenne.

Fumure :

Néant.

Prélèvement:

Après florasson. Foin.

\section{Pueraria phaseolides.}

Provenance :

Centre de Recherches Zootechniques de Miadana.

Nature du sol:

Colluvions (sol bien minéralisé).

Fumure :

Organique ( $30+$ de fumier par hectare).

Age de la plantation :

5 ans.

Prélèvement :

En fin de saison sèche. Séchage à l'ombre. 
Pueraria Thunbergiana.

Provenance:

Centre de Recherches Zootechniques de Kianjasoa.

Nature du sol:

Sol ferrallitique de plateau, de valeur moyenne.

Fumure :

Néant.

Récolte :

Végétation de saison des pluies. Séchage à l'ombre.

Desmodium infortum.

Provenance :

Centre de Recherches Zootechniques de Kianjasoa.

Culture :

Sur plateau,

Fumure:

Organique ( 30 t de fumier par hectare).

Récolte :

Végétation de saison des pluies. Foin.

\section{Phaseolus atropurpureus.}

Provenance :

Centre de Recherches Zootechniques de Miadana.

Nature du sol :

Colluvions (sol bien minéralisé).

Fumure :

Organique ( 30 t de fumier par hectare).

Age de la plantation :

3 ans.

Récolte :

Végétation de saison des pluies. Foin.

\section{Centrosema pubescens.}

Provenance :

Centre de Recherches Zootechnıques de Kianjasoa.

Nature du sol :

Sol ferrallitique de plateau, de valeur moyenne.
Fumure :

Organique ( 30 t de fumier par hectare).

Age de la plantation :

5 ans.

Prélèvement :

En saison des pluies. Séchage à l'ombre.

Glycine jovanica.

Provenance :

Centre de Recherches Zootechniques de Kianjasoa.

Nature du sol:

Sol ferrallitique de plateau, de valeur moyenne.

Fumure :

Organique.

Age de la plantation:

5 ans.

Prélèvement :

En saison des pluies. Foin.

Albizzia lebbek.

Provenance :

Centre de Recherches Zootechniques de Miadana.

Prélèvement :

Feuilles et jeunes tiges (correspondant aux parties mangées par les animaux) coupées sur des arbres de plus de 3 ans.

Prélèvement effectué en saison des pluies. Séchage à l'ombre.

Interprétation des résultats.

Nous nous bornerons à faire quelques remarques sur les teneurs trouvées pour les acidesaminés soufrés ainsi que pour l'iso-leucine et la lysine.

L'examen du tableau II fait ressortir un déficit important en cystine par rapport à la luzerne, pour toutes les légumineuses analysées, surtout pour le Vigna sinensis ( -47 p. 100), le Desmodium intortum (-41) et le Soja hispida ( -37$)$.

Par contre, toutes les teneurs en méthionine sont légèrement supérieures à celle de la luzerne (de +19 p. 100 pour le Pueraria phaseolides, Pueraria Thunbergiana et Saja hispida, d̀ + 2 p. 100 pour l'Albizzia lebbek). 
En ce 'qui concerne l'isoleucine, toutes les teneurs sont, dans l'ensemble, plus faibles que celle de la luzerne (de - 24 p. 100 pour le Soja hispida, à - 6 p. 100 pour le Vigna sinensis et -2 p. 100 pour le Pueraria phaseolides).

Le déficit en lysine de toutes les légumineuses fourragères analysées est beaucoup plus important, puisque, par comparaison avec la teneur de la luzerne, il s'exprime par : - 41 p. 100 pour le Vigna sinensis, -37 p. 100 pour le Pueraria phoseolides, - 31 p. 100 pour le Stylosanthes gracilis, et -30 p. 100 pour le Soja hispida.

\section{CONCLUSION}

Nous remarquons d'abord que, dans l'ensemble, la composition en acides-aminés présente une assez grande homogénéité pour les protides de toutes les légumineuses fourragères analysées.

Par rapport à la luzerne, nous constatons dans l'ensemble un taux nettement plus bas en lysine, et en cystine. Le déficit est plus faible pour l'iso-leucine. Par contre, les teneurs en méthionine sont légèrement plus élevées.

Nous ne manquerons pas de souligner les résultats particulièrement bons en ce qui concerne l'Albizzia lebbek. C'est là un argument de plus en faveur de l'utilisation des feuillages d'arbres qui peuvent être distribués à toutes les espèces.

Il est important enfin de situer les légumıneuses fourragères dans le contexte de l'alimentation des porcs ef des volailles d̀ Madagascar : les aliments de base disponibles sont constitués essentiellement par le manioc, le maiss, les issues de riz, les tourteaux d'arachide, et parfois, des farines de sang.

Si l'alimentation de base est le manioc, dont la carence azotée est bien connue, l'intérêt de l'utilisation des légumineuses est évident. Mais on peut prévoir qu'à elles seules, elles ne pourront assurer l'équilibre des acides-aminés, en raison notamment de leur insuffisance en cystine et méthionine.

Si la base alımentaire est le mais, les légumineuses seront utiles, car elles pourront pallier. au moins en partie, le déficit en lysine des grains.

Par comparaison avec la composition des issues de riz, on constate que les parties aériennes des légumineuses n'apportent pas d'acide-aminé souhaitable, mais elles sont presque équivalentes pour la fourniture des amino-acides indispensables, sauf pour les acides-amınés soufrés.

Nous avons dit plus haut que la culture des léğumineuses était envisagée pour une substi.tution partielle du tourteau d'arachide des rations. La présence d'aflatoxine oblige en effet souvent à limiter le taux de ce produit. L'examen de la composition en acides-aminés des légumineuses montre que cette substitution est tout d̀ fait valable, et serait même plus avantageuse en ce qui concerne la méthionine et la lysine, dont les. teneurs sont, dans l'ensemble, légèrement supérieures à celles du tourteau d'arachide.

En ce qui concerne la farine de sang, les légumineuses peuvent pallier en partie le déséquilibre iso-leucine/leucine de cet aliment, mais elles. ne peuvent prétendre le concurrencer pour l'apport de lysine.

En résumé, nous pouvons conclure que les parties aériennes des légumıneuses fourragères représentent une excellente source de protéines. végétales qu'un apport de méthıonıne pure dans. les rations peut élever à une valeur biologique correcte. Mais elles ne peuvent concurrencer les farines animales (et particulièrement les bonnes farines de poisson) pour un apport important de lysine.

Fournıssant à bas prix des protéines de qualité correcte, et apportant également des sels minéraux et des carotènes en quantıté, les légumineuses fourragères sont à conseiller à Madagascar en vert et sous forme de farines pour I'alimentation des porcs et des volailles. Il convient enfin d'ajouter qu'elles constituent également une excellente base d'alimentation pour les vaches laitières.

\section{REMERCIEMENTS}

Nous tenons à adresser à M. SERRES, Directeur de la Région de Recherches de Madagascar, nos vifș remerciements pour les précisions et les conseils concernant les problèmes de Nutrition, qu'il nous a apportés pour la rédaction de notre article.

Institut d'Elevoge

et de Médecıne vétérinatre des Pays tropicaux.

Laboratoire centrol de l'Elevage de Tonanarive (Madogascar). 


\section{SUMMARY}

Amino acids content of the principal leguminous of Madagascar

Amino acids content of the principal fodder leguminous of Madagascar has been recorded by hydrolisis; separation by chromatography on column of resine, and stained reaction with ninhydrine.

Tryptophane has been titreted according the colorimetric method of Fisch. The following fodder leguminous plants have been analysed :

- Vigna sinensis :

- Soja hispida ;

- Stylosonthes gracilis ;

- Pueraria phoseolides ;

- Pueraria Thunbergiana;

- Desmodium intortum;

- Phaseolus alropurpureus ;

- Centrosema pubescens :

- Glycine jovonica ;

- Albizzia lebbek.

Results are showned in tables.

\section{RESUMEN}

Composición en ácidos amınados

de las principales leguminosas forrajeras de Madagascar

Se determinó la composición en ácidos aminados de las principales leguminosas forrajeras de Madagascar después de hidrolisis, separación por cramatografia sobre columna de resina, y reacción coloreada con ninhidrına.

Se dosó el triptofonia mediante el método colorimetrico de Fischl.

Se analizaron las legumınosas siguientes :

- Vigno sinensis ;

- Soja hispida ;

- Stylosanthes gracilis ;

- Puerorio phaseolides;

- Puerorio Thunbergiana:

- Desmodium intortum;

- Phaseolus atropurpureus :

- Centrosema pubescens ;

- Glycine javonico ;

- Albizzia lebbek.

Los resultados estan notados en cuadros.

\section{BIBLIOG RAPHIE}

1. ADRIAN (J.) et RERAT (A.). - Méthodes d'Evaluation de la valeur nutritive des protéines (Les Cahiers Techniques du Centre National de Coordination des Etudes et Recherches sur la Nutrition et I'Alimentation C. N. R. S. 1958).

2. CAPITAINE (P.), GRANIER (P.), GAULIER (R.), GILIBERT (J.) et DUBOIS (P.). — Amélioration de l'alimentation du bétail à Mada- gascar : Le Pueraria phaseolides « Kudzu». Rev. Elev. Méd. Vét. Poys Trop. (sous presse). 3. GRANIER (P.) et LAHORE (J.). - Amélioration de l'alimentation du bétail à Madagascar : Amélioration économique du pâturage naturel par le Stylosanthes gracilis. Publié par l'Inst. Elev. Méd. Vét. Pays Trop. Région de Recherches de Madagascar. Avril 1966. 
4. INGLIS (A. S.) et LEAVER (I. H.). - Studies in the determination of tryptophan. Modified FISCHL procedure. Analytical Biochemistry, 1964, 7, 1, 10 à 17.

5. MOORE (S.) et STEIN (W. H.). - Chromatography of Amino-acids on sulfonated polystyrene resins. Journal of Biological Chemistry, 1951, 192, 663-681.

6. MOORE (S.), SPACKMAN (D.) et STEIN (W. H.). - Chromatography of Amino-Acids on sulfonated polystyrene resins. Analytical Chemistry, 1958, 30, 1185 et sq.

7. MOORE (S.) et STEIN (W. H.), - A modified ninhydrin reagent for the photomeiric determination of amino-acids and related compounds. Journal of Brological Chemistry, 1954, 211, 907-913.

8. SERRES $\left(H_{.}\right)$. - Eléments d'alimentation du bétail à Madagascar. Publié par l'Inst. Elev. Méd. Vét. Pays Trop. Région de Recherches de Madagascar - 1966.

9. SCHRAM (E.), MOORE (S.) et BIGWOOD (E. J.). - Chromatographic determination of cystine as cysteic acid. Biochemical Journal, 1954, 57, 33-37.

10. VAILLANT $\left(R_{1}\right)$, - Les aliments du bétail à Madagascar. Publié par l'Imprimerie Officielle de Tananarive 1957. 